\title{
Afrontamiento y viudez en la vejez: Estudio mixto en población colombiana
}

\section{Coping and widowhood in the older adulthood:}

Mixed methods study in Colombian population

María Fernanda Reyes-Rodríguez ${ }^{1}$, María-Angélica Cujiño-Medrano ${ }^{2}$,

Daniela Cano-Santana ${ }^{3}$, Sonia Orozco-Barragán ${ }^{4}$, Ángela Pereira-Morales ${ }^{5}$

y María Camila Díaz-Trujillo

${ }^{1} \mathrm{PhD}$. en Psicogerontología, MSc. en Metodología de la Investigación. Profesora asociada de la Facultad de Psicología de la Universidad El Bosque, Bogotá, Colombia.

E-mail: reyesmariafr@unbosque.edu.co

${ }^{2}$ MSc. en Psicología Clínica. Profesora asociada de la Facultad de Psicología de la Universidad

El Bosque, Bogotá. E-mail: cujinomaria@unbosque.edu.co

${ }^{3}$ Psicóloga. Auxiliar de investigación en la Universidad El Bosque, Bogotá.

E-mail: dcano@unbosque.edu.co

${ }^{4}$ Psicóloga. Auxiliar de investigación en la Universidad El Bosque, Bogotá.

E-mail: sorozco@unbosque.edu.co

${ }^{5}$ Psicóloga. Auxiliar de investigación en la Universidad El Bosque, Bogotá.

${ }^{6}$ Psicóloga. Auxiliar de investigación en la Universidad El Bosque, Bogotá.

E-mail:kamila038@hotmail.com

Facultad de Psicología, Universidad El Bosque.

Bogotá, Colombia.

\section{Resumen}

La viudez está catalogada como uno de los eventos de vida adversos más estresantes. El cambio de estado civil genera un cambio de rol e implica una resignificación de la vida. El presente estudio tuvo como objetivo comprender la forma en que las personas mayores viven y afrontan la viudez y determinar cuál es la relación entre las respuestas psicológicas, el sexo y los estilos de afrontamiento. Se utilizó metodología mixta con un diseño CUALI-cuanti a partir de la teoría fundamentada y análisis log lineal. La muestra estuvo compuesta por 29 personas mayores viudas residentes en Bogotá. Este estudio reporta las respuestas psicológicas de un grupo de personas mayores viudas que facilitan el afrontamiento exitoso a la pérdida de la pareja y aquellas que lo dificultan. Los resultados en la fase cualitativa mostraron que el afrontamiento a la viudez se enmarca en siete factores: sentido de vida, apoyo social, relación previa, satisfacción con el matrimonio, participación en actividades, consecuencias generadas por la pérdida y fortalezas personales. En la fase cuantitativa se encontró una interacción significativa entre respuesta psicológica y afrontamiento, lo que reveló que algunas respuestas psicológicas en parti- 
cular llevan a un afrontamiento activo o por el contrario, lo dificultan. Las respuestas que se encontraron significativas fueron aislamiento, soledad, seguir adelante y participación en actividades. Los resultados demuestran que el afrontamiento es producto de las interacciones entre los sistemas macro y micro y muestra a la viudez como una transición multifacética. Se discuten las similitudes con estudios anteriores y limitaciones.

Palabras clave: viudez; afrontamiento; vejez; metodología mixta; respuesta psicológica.

\section{Abstract}

Widowhood is cataloged as one of the most stressful adverse life events; the change of civil status generates a change of role and implies a resignification of life. The aim of this study was to understand how older people experience and cope with widowhood. The research used mixed methods approach with a QUALI- quanti design, through grounded theory and log lineal analysis, with a sample of 29 elderly widows and widowers living in Bogotá. This study reports the psychological responses of a group of elderly widows that facilitate successful coping with the loss of the couple, and the responses that make it difficult. The results in the qualitative stage showed that coping with widowhood is framed in seven factors: sense of life, social support, previous relationship, satisfaction with marriage, participation in activities, consequences generated by the loss and personal strengths. In the quantitative stage a significant interaction between psychological response and coping was found, which revealed that some psychological responses in particular lead to an active coping or, on the contrary, made coping difficult. The answers that were significant were isolation, loneliness, moving forward and participation in activities. The results revealed that coping is a product of the interactions between macro and micro systems and showed widowhood as a multifaceted transition. Similarities with previous studies and limitations are discussed. Keywords: widowhood; coping; aging; mixed methods; psychological responses.

\section{Introducción}

La pérdida del cónyuge es considerada uno de los sucesos de vida más estresantes, especialmente en la vejez (Bennett y Soulsby, 2012; Carr, Nesse y Wortman, 2006) en donde existe una alta probabilidad de experimentarla (Bonnanno y Kaltman, 1999). La pérdida de la pareja parece tener efectos importantes en el bienestar en la adultez mayor porque trae consigo nuevos desafíos y puede generar desequilibrio psicológico (Bennett, Hughes y Smith, 2005). Existe evidencia de que la viudez es un factor de riesgo de mortalidad, especialmente en los hombres (Stimpson, Kuo, Ray, Raji y Peek, 2007). Esto se debe a que los hombres tienden a depender principalmente de su pareja para el apoyo instrumental y emocional, mientras que las mujeres mantienen vínculos sociales más amplios (Bennett, 2006; Carr, 2004; Williams y Umberson, 2004).

Contar con una pareja hace más fácil sobrellevar situaciones económicas difíciles (Antonucci et al., 2002; Dupre y Meadows, 2007), la pobreza agudiza todas las causas de mortalidad, pero sus efectos difieren según el estado civil (Choi y Marks, 2011; Dupre y Meadows, 2007). Las mujeres mayores que dependen económicamente de los hombres parecen tener más dificultades en el afrontamiento, que quienes son más independientes económicamente durante el matrimonio (Ángel, Jiménez y Ángel, 2007; Bennett, 2010).

La pérdida de la pareja demanda a que las personas utilicen todas sus fuerzas y habilidades para restablecer sus vidas, esto comúnmente se ha estudiado desde el proceso de duelo. Otro enfoque ha sido el estudio de la viudez separada del duelo, la cual se entiende como un cambio de la condición de casado al nuevo rol de ser viudo, lo que implica una reconstrucción de identidad. Es por esto que es 
fundamental comprender cómo es el proceso de afrontamiento y los efectos a largo plazo de la pérdida (Bennett et al., 2005; Wilcox et al., 2003).

Existen diversos factores que influyen en el afrontamiento a la pérdida y adaptación a la viudez. Por ejemplo, cuando la muerte del cónyuge es considerada como un reto favorece el desarrollo personal y facilita el afrontamiento (Bennett y Vidal, 2000; Bennett, Hughes y Smith, 2003; Bonnano, Wortman y Neese, 2004). El apoyo social se ha encontrado como facilitador de un afrontamiento exitoso siempre y cuando sea constante (Bisconti, Bergeman y Boker, 2006; Wruz, Hänel, Wagner y Neyer, 2013). En la viudez la fuente de apoyo más común son los hijos, aunque se han identificado otras como hermanos, amigos o vecinos (Maher, Mora y Leventhal, 2006; Silverstein y Giarrusso, 2010).

Se han encontrado diferencias en el tipo y fuente de apoyo entre hombres y mujeres; las mujeres reciben más apoyo de sus hijos y el tipo de apoyo es económico o instrumental, lo que está relacionado con roles culturales (Guiaux, Van y Broese, 2007). En el caso de los viudos es común que el apoyo de los hijos sea de tipo instrumental o emocional (Antonucci et al., 2002; Ha, Carr, Utz y Nesse, 2006). La viudez afecta de manera importante a los hombres (Bennett, 2003), debido a su dependencia en las tareas del hogar, así como por las relaciones de poco apego con familiares cercanos (Ocampo, Romero y Saa, 2001; Sánchez, 2009), lo que hace que perciban sentimientos de soledad en la viudez (Carr et al., 2006). También se sabe que es más probable que los hombres establezcan nuevas relaciones de pareja y que tras la pérdida sean más activos sexualmente que las mujeres (Karraker y De Lamater, 2013), en tanto que las mujeres tienden a realizar más actividades sociales que los hombres como voluntariados, lo que ayuda a mejorar la autoeficacia y la autoestima y a establecer nuevas relaciones sociales (Holm y Severinsson, 2012; MorrowHowell, 2010). Adicional a ello se ha encon- trado que no solo es importante el apoyo de las redes cercanas en la viudez, sino que también el apoyo del gobierno es fundamental. Un estudio transcultural realizado en Estados Unidos, Francia, Alemania y Japón encontró que el apoyo del estado y las políticas gubernamentales, en especial el apoyo económico, influye significativamente en el afrontamiento exitoso a la viudez (Antonucci et al., 2002).

Otros factores influyen en el afrontamiento a la pérdida de la pareja en la adultez mayor, por ejemplo, tener un sentido de vida (Welsh, Moore y Getzlaf, 2012), autoeficacia (Fry, 1998) y espiritualidad (Tak-Cheng y Chan, 2006), la cual está relacionada a la satisfacción de vida tanto en viudas como en viudos. Un factor adicional es la calidad del matrimonio antes de la pérdida. Por ejemplo, (Carr et al., 2000) encontraron que el ajuste a la viudez era más difícil para los que experimentaron mayores niveles de calidez emocional $\mathrm{y}$ dependencia instrumental en su matrimonio, mientras que personas viudas que reportan mayor calidad marital muestran un mayor incremento en el número de síntomas de depresión que aquellos con baja calidad marital (Schaan, 2013).

En el contexto latinoamericano, se realizó un estudio en Perú en el que se encontró que la pérdida del cónyuge es uno de los eventos de más difícil adaptación, especialmente para los hombres (Del Risco, 2009). En Cuba, Pérez (2008) halló que la pérdida de la pareja resulta ser un factor determinante del cese en la actividad sexual en esta etapa de la vida. En Chile, Vega (2009) encontró que hay cambios positivos tras la pérdida en la adultez mayor, principalmente en las mujeres, debido a que experimentan independencia y libertad.

El presente estudio se basa en la investigación mixta realizada por Bennett et al. (2005), quienes examinaron las respuestas psicológicas de un grupo de personas mayores viudas residentes del Noroeste de Inglaterra. Encontraron que es posible identificar respuestas psicológicas que permiten distinguir entre los viudos y viudas que logran afrontar exitosa- 
mente y quiénes no. A través de un análisis log-lineal, hallaron una interacción significativa entre afrontamiento, sexo y respuesta psicológica, lo que evidenció que una respuesta en particular lleva a un afrontamiento exitoso de manera diferente en los hombres que en las mujeres. Por ejemplo, la respuesta psicológica de soledad resultó ser específicamente de las mujeres que lograron afrontar la pérdida, mientras que la respuesta codificada como egoísmo -que hace referencia a una toma de decisiones para la propia satisfacción, para liberarse de la carga y obligación que implica el matrimonio- caracterizó a los hombres que afrontaron exitosamente la pérdida. Por otro lado, reportaron una interacción significativa entre afrontamiento y respuesta psicológica. Quienes lograron afrontar exitosamente la pérdida presentaron la respuesta psicológica de hablar con el cónyuge muerto, mientras que el ensimismamiento fue una respuesta relacionada con quienes no la afrontaron. Bennett et al. (2005) clasificaron el afrontamiento exitoso como positivo o activo (Carver, Scheier y Weintraub, 1989). El presente estudio se basa en esta conceptualización de afrontamiento de Carver et al. (1989) quienes definen el afrontamiento activo como las respuestas psicológicas adaptativas que junto con altos niveles de optimismo llevan a la persona a lograr un afrontamiento activo y centrado en el problema. Este tipo de afrontamiento se ha relacionado con un funcionamiento más adaptativo y una reducción de las secuelas psicológicas. Determinadas estrategias de afrontamiento activo son usualmente descritas como adaptativas, mientras que aquellas denominadas pasivas, consisten en la ausencia de enfrentamiento o conductas de evitación que son consideradas desadaptativas (Carver et al., 1989).

El presente estudio tuvo como objetivo comprender la forma en que las personas mayores viven y afrontan la viudez, y determinar cuál es la relación entre las respuestas psicológicas, el sexo y los estilos de afrontamiento en una muestra de personas mayores viudas en Colombia. La investigación se basó en el estudio mixto realizado por Bennett et al. (2005).

\section{Método}

Se realizó un estudio a través de metodología mixta para comprender cómo las personas mayores viudas viven y afrontan la viudez. Con el enfoque mixto se buscó recoger y analizar datos, para extraer conclusiones a través de los métodos cualitativos y cuantitativos en un mismo estudio (Cresswell, 2014; Tashakkori y Cresswell, 2007).

El estudio utilizó un diseño de dos etapas (CUALI- cuanti). La etapa cualitativa buscó responder a la pregunta ¿cómo viven y afrontan la viudez, un grupo de 29 personas mayores residentes de Bogotá? a través de teoría fundamentada. La etapa cuantitativa se enfocó en la categoría de respuesta psicológica según el éxito en el afrontamiento y sexo. En esta etapa se buscó responder a una segunda pregunta: ¿Cuál es la relación entre las respuestas psicológicas y los estilos de afrontamiento? ¿Es igual en hombres que en mujeres que han enviudado? a través de un análisis log-lineal.

\section{Participantes}

El grupo de participantes estuvo conformado por 29 personas mayores viudas (13 hombres y 16 mujeres) entre 55 y 90 años de edad $(M=74)$. Todos los participantes habían tenido la pérdida de la pareja en un tiempo superior a seis meses, debido a que nuestro interés era entender la viudez separada del duelo. De las 29 personas mayores viudas participantes, se encontró que 20 presentaron un afrontamiento activo al momento de la entrevista y que nueve no cumplían los criterios de afrontamiento activo, por lo que fueron clasificados como afrontamiento pasivo o no afronta. Los participantes fueron clasificados según el procedimiento seguido por Bennet et al. (2005), el cual se basa en los criterios de 
afrontamiento activo y pasivo de Carver et al. (1989). Con base en los códigos identificados, en la fase cualitativa se ubicaron las respuestas según reflejaban afrontamiento activo o pasivo. En afrontamiento activo se ubicaron aquellas personas que en su relato mostraban estrategias de afrontamiento centradas en el problema, presentaban un funcionamiento adaptativo, habían logrado retomar sus actividades previas y no manifestaban percibir secuelas psicológicas asociadas a la pérdida. En el afrontamiento pasivo se clasificaron personas que según su discurso mostraban ausencia de enfrentamiento y conductas de evitación, así como la presencia de dificultades para seguir adelante con la vida. Estas personas manifestaban no tener un sentido de la vida, parecían no haber retomado sus actividades previas o algunas nuevas y aseguraban no poder sobrellevar la pérdida (Bennett et al., 2005; Carver et al., 1989).

\section{Técnicas de recolección de datos}

\section{Entrevista semiestructurada}

La entrevista se basó en el trabajo realizado por Bennett et al. (2005). La parte inicial estaba dirigida a realizar una introducción sobre el estudio y conocer los aspectos sociodemográficos de los participantes, tiempo de viudez, duración del matrimonio y relaciones familiares y aspectos generales relacionados con la vida actual de viudo/a. Después la entrevista se encaminaba a explorar cómo había sido la relación de la pareja y su vida durante el matrimonio. La siguientes secciones se enfocaron en comprender cómo las personas afrontaron la pérdida a través de: (a) exploración del momento de la pérdida, (b) exploración después de la muerte del cónyuge (no se enfatizó en el duelo, se indagó si la vida cambió y de qué forma), (c) exploración sobre cómo era su vida al momento de la entrevista y (d) se profundizó sobre los eventos que le ayudaron a afrontar la viudez.

La exploración de estas categorías permitió determinar los estilos de afrontamiento de los participantes (activo o pasivo) y las respuestas psicológicas. A continuación se definen las categorías.

\section{Afrontamiento.}

Se entiende como cualquier respuesta, tanto emocional como comportamental que el individuo lleva a cabo para sobrellevar la pérdida de su pareja y la viudez. Afrontamiento activo hace referencia a las respuestas psicológicas adaptativas que junto con altos niveles de optimismo llevan a la persona a lograr un afrontamiento activo y centrado en el problema. Este tipo de afrontamiento se ha relacionado con un funcionamiento más adaptativo y una reducción de las secuelas psicológicas que las situaciones adversas traen consigo (Carver, Scheier y Weintraub, 1989). Afrontamiento pasivo Esta consiste en la ausencia de enfrentamiento y conductas de evitación, así como, la presencia de dificultades para seguir adelante con la vida. Estas personas manifiestan no tener un sentido de la vida, parecen no haber retomado sus actividades previas o algunas nuevas y aseguran no poder sobrellevar la situación (Carver, Scheier y Weintraub, 1989).

\section{Respuesta psicológica.}

Bennett, Hughes y Smith (2005) la definen como la forma en que las personas responden a la viudez; se compone de los comportamientos y las emociones producidos como consecuencia de la pérdida. Esta definición abarca las nociones de los procesos de afrontamiento y la eficacia de las respuestas, adicionalmente tiene en cuenta el papel de los recursos personales, sociales y ambientales.

\section{Procedimiento y análisis de datos}

La investigación inició con la etapa cualitativa, primero se realizó un entrenamiento a auxiliares de investigación, todas las autoras del artículo participaron en la realización de las entrevistas. Después del entrenamiento, 
se inició con la consecución de participantes, quienes fueron contactados a través de líderes de grupos de personas mayores y referidos. Las personas invitadas a participar fueron informadas sobre el objetivo del estudio, la voluntariedad y la confidencialidad en la participación. Los interesados dieron su consentimiento. Todas las entrevistas fueron grabadas en audio y transcritas. Para cada entrevista se recolectaron unas notas de campo, las cuales se tuvieron en cuenta para el análisis.

Se utilizó el enfoque de teoría fundamentada (grounded theory) que es un método sistemático, inductivo, comparativo y flexible para la recolección y análisis de datos cualitativos para la construcción de teoría (Bryant y Charmaz, 2007; Strauss y Corbin, 2002). El análisis se realizó con base en la propuesta de Charmaz (2006) para la lectura y codificación de las entrevistas. Como lo propone la teoría fundamentada, este método fue adoptado sin tener ninguna suposición ni categoría preconcebida. Primero las entrevistas fueron leídas y re-leídas para tener un entendimiento general de las experiencias y luego se realizó la codificación inicial de las mismas, línea por línea. Este proceso fue abierto y cercano a los datos, es decir, los datos no se exploraron desde categorías preconcebidas. Seguidamente, se realizó una codificación enfocada en donde se seleccionaron códigos analíticos por cada participante, identificándose 361 códigos axiales. Al finalizar esta codificación, las entrevistas fueron intercambiadas entre las autoras con el fin de aumentar la confiabilidad en el análisis.

Se continuó con la codificación axial, en la cual se pretendía buscar relaciones entre categorías y subcategorías, lo que generó la conceptualización de estas y la creación de la teoría sustantiva. Es de resaltar que durante el análisis se formularon preguntas a las que se les fue dando respuesta y las categorías de la teoría fueron elaboradas en consenso. Adicionalmente, se realizó la clasificación de los participantes que tenían un afrontamiento activo o pasivo según el procedimiento realizado por Bennett et al. (2005).

Inicialmente cada entrevista y sus códigos fueron leídos y los aspectos tanto verbales como no verbales se usaron para la clasificación de los participantes. Esta clasificación fue realizada de manera individual y después discutida en el grupo de investigadores. Por ejemplo, aquellos participantes que manifestaron específicamente estar teniendo dificultades para afrontar la pérdida, o fue evidente durante la entrevista que experimentaban angustia y malestar al hablar de la pérdida, fueron clasificados como afrontamiento pasivo. Las personas que fueron clasificadas como afrontamiento activo reflejaban, tanto en la entrevista como sus códigos, que habían logrado continuar y adaptarse a su nueva vida sin su cónyuge así como hablar sobre la pérdida sin demasiada angustia o dolor.

Por último, se realizó el análisis cuantitativo de los datos por medio del análisis log-lineal, el cual permite evaluar asociaciones entre varias variables categóricas. Inicialmente se buscó clasificar los códigos resultantes del análisis con teoría fundamentada, por medio de frecuencias, para determinar el tipo de interacción entre lasvariables sexo, respuesta psicológica y afrontamiento. Los códigos pertenecientes a las interacciones significativas permitieron la clasificación en afrontamiento o no afrontamiento. Posteriormente se realizó la fase de modelado, en la que se sometieron a prueba los diferentes modelos posibles, derivados de la fase anterior y se seleccionó el más conveniente, que reuniera requisitos de parsimonia y bondad de ajuste. El procedimiento de ajuste se realizó calculando el estadístico $\chi^{2}$ y evaluando su significación estadística. Por último, se llevó a cabo la fase de diagnóstico y evaluación, en donde se identificaron interacciones significativas entre las variables (sexo, afrontamiento y respuesta psicológica). 


\section{Resultados}

\section{Pregunta 1: ¿Cómo vive y afronta la viudez un grupo de 29 personas mayores residentes de Bogotá?}

Los resultados muestran que el afrontamiento a la viudez se enmarca en siete factores. Se hallaron dos factores transversales, sentido de vida y apoyo social, los que son alimentados por cinco factores: (a) relación previa, (b) satisfacción con el matrimonio, (c) participación en actividades, (d) consecuencias generadas por la pérdida y (e) fortalezas personales.

\section{Sentido de vida}

El sentido de vida se refiere a tener una motivación para seguir adelante con la vida. Se encontró que las personas que tenían sentido de la vida lograron un afrontamiento activo y en aquellas que no lo tenían, su afrontamiento se vio afectado.

Las personas que no lograron afrontar de manera activa, la muerte del cónyuge significó una pérdida de sentido de vida, como lo afirma $\mathrm{AB}$ (mujer, 85 años, no afronta) “... alegría no es mucha, yo no vivo muy feliz, feliz no vivo yo, desde que él murió, feliz, feliz, no soy, así esté con todos mis hijos, esté en paseos y todo, no". Las personas que no tienen un sentido de vida presentaron sentimientos de soledad y anhelo, guardan las pertenencias intactas, se aíslan y hablan con el cónyuge muerto. Por ejemplo, AB (mujer, 85 años, no afronta) afirma “...yo siempre quise soñarme con él, yo me siento aquí (sala), y yo me acuerdo que muchas veces estaba yo aqui (mueble de la sala) y él bajaba, y yo empiezo a llamarlo, le digo: ay $H$, por ahi ven, ven me acompañas...". La respuesta psicológica de aislamiento se refleja en la afirmación de MA (mujer, 56 años, afrontamiento pasivo): "yo me encerraba, me la pasaba muchas veces sola, no me llamaba la atención arreglarme o vestirme o tener algo diferente, sino ir a ence- rrarme”.

La respuesta de conservar las pertenencias intactas hasta la actualidad se relacionó con un afrontamiento pasivo y es una manifestación de anhelo. Como es el caso de $\mathrm{AB}$ (mujer, 85 años, no afronta): "conservo intacto el escritorio de trabajo y las placas de reconocimiento de él porque me parece bonito recordarlo así (...); por la misma razón sigo en la misma casa donde viviamos juntos, mis hijos me decían que me fuera, pero no lo hago porque es una manera más de recordarlo, vaya donde vaya siempre estará el recuerdo de él".

\section{Relación de pareja}

La relación de pareja durante el matrimonio influye en la forma en que los participantes viven y afrontan la viudez. Especialmente, la satisfacción con el matrimonio, el tipo de rol y el mantenimiento de este después de la pérdida, así como la dependencia instrumental o económica de su pareja.

\section{Satisfacción con el matrimonio.}

Esta categoría se entiende como la evaluación que hacen los participantes de la satisfacción con su relación afectiva. Las personas que evaluaron su relación como satisfactoria compartían actividades placenteras para ambos y mantenían un ambiente agradable en su hogar. En algunos casos se refleja que la satisfacción con el matrimonio facilitó un afrontamiento activo, porque evaluaban su relación de pareja y matrimonio como una meta de vida cumplida. Un ejemplo de esto es la afirmación de PR (hombre, 77 años, afronta): "bendito sea el Señor, yo doy gracias al Señor por haberme permitido conseguir una señora que me supo entender, la pasamos mиу тиу тиу felices el tiempo que duramos".

Ahora bien, el sentirse insatisfecho con la relación matrimonial, fue un factor que se relacionó con el éxito en el afrontamiento a la pérdida, pues esto hizo que, especialmente en las mujeres, las personas tuvieran un descanso 
y sensación de libertad después de la pérdida. CB (mujer, 56 años, afronta): “Al principio la vida juntos fue muy buena, estuvimos, disfrutamos mucho pero ya después él cayó en las drogas y ya la vida cambió rotundamente.... Entonces descansé porque como él estaba metido en el mundo de las drogas, para mí fue un descanso". Adicionalmente, la pérdida de la pareja le dio a las mujeres autonomía e independencia económica, PAU (mujer, 65 años, afronta): "Eran muy independientes de mí porque yo no tenía los medios para atenderles una invitación, para hacerles un almuerzo, porque yo no tenía, pero ya como Dios me dio esa bendición de esa pensión (de su esposo), ahora sí comparto con mi gente".

\section{Roles.}

Se encontró que el mantenimiento de los roles resulta decisivo para el afrontamiento, ya que evita una ruptura severa en su cotidianidad e identidad. Por ejemplo, el rol de mujer activa y trabajadora se identificó como facilitador de un afrontamiento activo, puesto que la libertad de decisión e independencia económica que tuvieron durante su vida de casadas no se modificó tras a la muerte de su esposo, por lo tanto no tuvieron que adquirir esta habilidad. El rol de mujer trabajadora se define como aquella mujer que contribuyó con el sostenimiento económico del hogar, por ejemplo LNC (mujer, 77 años, afronta): "Yo trabajé 50 años en el Magisterio; trabajé en primaria, en primaria toda la vida, yo me licencié en sociales, tengo un título de licenciada en sociales y un postgrado en informática educativa... ¿Eso le ayudo a afrontar? ¡Claro! pues eso me ayudó a aumentar mi pensión porque hice mi licenciatura y el postgrado".

\section{Dependencia económica.}

Esta categoría solo se encontró en las mujeres, algunas de ellas necesitaron el apoyo económico de su cónyuge durante el matrimonio. Asumir la responsabilidad del manejo de la economía del hogar después de la pérdida garantizó un éxito en el afrontamiento para algunas mujeres. Las mujeres que asumieron el rol de manejo del dinero generaron independencia económica y sensación de libertad. Por ejemplo: AU (mujer, 65 años, afronta): “...pero a la larga yo triunfé, ¿por qué? dirá usted, porque cuando él falleció yo comencé como a asimilar la situación, la vida, a disfrutar de lo que él me dejó, con mis hijos, al que yo pueda ayudarle le ayudo...".

Por el contrario, las mujeres que no suplieron esta necesidad carecían de apoyo de sus hijos o de personas, como familiares y amigos cercanos, o no podían continuar con el trabajo por problemas de salud, por lo cual no lograron el afrontamiento como es el caso de AC (mujer, 72 años, afrontamiento pasivo) comenta: "Diosito santo, qué hago que estoy tan triste, estoy tan ay, yo no esto, porque a mi me toca todo, que pagar servicios, que darles de comer".

\section{Dependencia instrumental.}

Algunos participantes dependían de sus parejas para el desarrollo de actividades relacionadas con el cuidado personal y oficios domésticos. GG (hombre, 65 años, afronta) comenta: "Pasan los meses en los que echa a sentir o hacerle falta lo que ella hacía por uno, es cuando uno empieza a sentir el dolor duro y como ella me hacía comida, me recogía interiores...". Algunos participantes intentaron suplir esta necesidad con una nueva relación de pareja o aprendiendo a realizar estas actividades, lo que influyó en el afrontamiento exitoso. La búsqueda de una nueva pareja se encontró especialmente en los hombres, lo que ayuda a suplir necesidades instrumentales y emocionales, por ejemplo GG (hombre, 65 años, afronta): "Ella de pronto se enamoró de mí y después me enamoré yo, duramos 6 años. Tenía 7 años de viudo".

\section{Apoyo social}

El apoyo social es determinante en todo el proceso de muerte, duelo y viudez. Los resul- 
tados mostraron que el contar con compañía de manera inmediata después de la muerte facilitó el afrontamiento a la pérdida. FO (mujer, 80 años, afronta) afirma: "Estuve acompañada con una de mis hijas que vino, ehhh... pues como acompañada con la familia en general que estaban pendientes de mi y quizá eso pues me hizo llevar las cosas pues, no fácil pero sí en mejores condiciones". La ausencia de todo tipo de apoyo social afecta severamente el afrontamiento. AC (mujer, 72 años, no afronta): "Entonces ya uno ve el vacio tan grande y como yo no tengo amigas no tengo a nadie, ni familia ni nada".

El apoyo social era brindado básicamente por familiares y amigos. Las personas más cercanas en el proceso de muerte, duelo y viudez fueron los hijos y familiares. RO (84 años, afronta) comenta: "Yo no me siento sola porque tengo nietos y bisnietos, hay gente que sí le toca solita”. En cuanto al apoyo de los amigos, se encontró que el tipo de apoyo es primordialmente emocional e instrumental, por ejemplo PR (hombre, 77 años, afronta): "Eso siempre, siempre le dan a uno ánimo, por ahí no falta que uno se caiga, y... ay venga, que le paso, lo ayudan, y eso es vida”.

\section{Religión}

Las creencias religiosas fueron evidentes en la mayoría de las personas mayores. Esta fe y religiosidad jugaron un rol importante en el afrontamiento, ayudó a darle sentido a la muerte al considerar que fue un plan de Dios. Respecto a esto, LNC (mujer, 77 años, afronta): "Yo me siento tranquila en cuanto a la falta de él, yo he superado eso muy bien, yo lo he asimilado muy bien, porque para mí tener que verlo en una cama, para mí eso no, fue lo mejor que pudo hacer mi Dios, así me toque bregar a mí, era lo que él quería, pues él siempre me decía: 'no, yo tengo que irme primero, yo no me siento capaz de quedarme aquí solo, no, que voy a hacer yo aquí solo?, no, yo tengo que irme primero' (risas), ¿es lo que generalmente sucede, no?, los hombres casi siempre mueren primero, es lo que mi Dios disponga".

A pesar de que la práctica religiosa se encontró como una fuente de esperanza y tranquilidad, en algunos casos esta práctica no fue suficiente ya que debe ir de la mano del apoyo social. Un ejemplo de esto es AG (mujer, 80 años, no afronta): "Cuando él murió yo asistí más seguido (a misa) porque ya no le pedía permiso a nadie y es lo único que yo tengo, ir a la Iglesia, porque yo no salgo a ningún otro lado, solo los domingos a la iglesia".

\section{Fortalezas personales}

Las características personales adaptativas influyen en el afrontamiento a la pérdida. Se encontró que algunas personas parecían tener rasgos de personalidad optimista y resiliente, lo que les ayudó a seguir adelante y no centrarse en el dolor. GG (hombre, 65 años, afronta): "De todas maneras uno tiene que olvidar, lo que ya fue, fue, la que se murió se murió, de todas maneras toca olvidar". Las personas que parecían optimistas lograban tener un estado de ánimo positivo a pesar de la pérdida. Por ejemplo, OC (hombre, 74 años, afronta): “...pues yo soy un hombre de 70 bien conservado, que no fuma, no trasnocha, se pega su acostadita pero nada más, además eso es saludable para el sistema cardíaco, jajaja, para el estrés”.

\section{Participación en actividades}

Las personas realizan nuevas actividades, donde se pueden distraer, aprender nuevos conocimientos o mantener sus roles. Esta categoría afecta positivamente especialmente si estas actividades son placenteras y los hacen sentir productivos. Por ejemplo, JMC (hombre, 73 años, afronta): “... el trabajo pues ha sido como la mejor terapia, la mejor, me mantiene muy distraído, todo el tiempo estoy acá en movimiento, atendiendo, resolviendo...".Y otros, simplemente buscan mantenerse ocupados. FO (mujer, 79 años, 
afronta) “ ¡No no no!, yo tengo el teléfono hasta del gato, por ejemplo, yo llamo a Neiva, a Barranquilla, yo llamo a Cartagena, Medellín... o hago programa o si no me lo invento (risas)...".La inactividad afecta gravemente el afrontamiento, ya que genera aburrimiento y promueve sentimientos de soledad y tristeza. MA (mujer, 56 años, no afronta):"ahorita en esta época eso es tremendo porque pasa sábado, domingo, lunes, martes, miércoles, sábado, domingo, y es lo mismo ¿sí?, no hay ninguna, nada diferente, sí, nada (llora)".

\section{Pregunta 2: ¿Cuál es la relación entre las respuestas psicológicas, sexo y estilos de afrontamiento en un grupo de personas viudas mayores de 55 años en Bogotá?}

Se encontró que de las 29 personas mayores viudas que participaron en la investigación, nueve no afrontaron ( 5 mujeres y 4 hombres) $\mathrm{y}$ que 20 presentaron un afrontamiento activo al momento de la entrevista (10 hombres y 10 mujeres). Se realizó un análisis log- linear para evaluar las posibles interacciones de tres vías entre afrontamiento, sexo y respuesta psicológica. Los resultados revelaron una interacción significativa entre respuesta psicológica y afrontamiento $\left(\chi^{2}=32.88, p=.001\right)$, las otras interacciones no se encontraron significativas (sexo*afrontamiento: $\chi^{2}=1.00, p=$ n.s.; sexo*respuesta: $\chi^{2}=1.66, p=$ n.s. $)$. Adicionalmente se encontró que afrontamiento $\left(\chi^{2}=\right.$ $6.04, p=.001)$ y tipo de respuesta $\left(\chi^{2}=32.96\right.$, $p=.001)$ fueron variables significativas para la clasificación, la variable sexo no se encontró significativa $\left(\chi^{2}=.86, p=\right.$ n.s. $)$.

El hallazgo de una interacción entre tipo de respuesta y afrontamiento revela que algunas respuestas psicológicas en particular llevan a un afrontamiento activo o por el contrario, lo dificultan. Las respuestas que se encontraron significativas fueron aislamiento, soledad, seguir adelante y participación en actividades (Ver Tabla 1).

Tabla 1

Respuestas psicológicas y afrontamiento.

\begin{tabular}{c|c|c}
\multicolumn{1}{c|}{ Interacción } & Respuesta psicológica & Afrontamiento \\
\hline \multirow{2}{*}{ Afrontamiento*Respuesta } & Aislamiento & Mujeres que no afrontan \\
Soledad & Mujeres que no afrontan \\
Seguir adelante & Mujeres que afrontan \\
& Participación en actividades & Hombres y mujeres que afrontan
\end{tabular}

Las mujeres que presentaron aislamiento y soledad tras la pérdida no lograron afrontar, aunque estas respuestas también dificultaron el afrontamiento de algunos de los hombres que las reportaron. En cuanto al afrontamiento activo, seguir adelante, fue una respuesta específica para las mujeres que afrontaron, pero no para los hombres. Sin embargo, la participa- ción en actividades sí fue determinante para que ambos lograran un afrontamiento activo. En la Tabla 2 se pueden observar las respuestas psicológicas que se relacionaron significativamente con un afrontamiento activo o pasivo si los hombres o mujeres lo reportaron en mayor o menor grado. 
Tabla 2

Tabla de contingencia del tipo de afrontamiento por respuesta y sexo.

\begin{tabular}{|c|c|c|c|}
\hline \multirow{2}{*}{$\begin{array}{l}\text { Respuesta } \\
\text { psicológica }\end{array}$} & \multirow{2}{*}{$\begin{array}{c}\text { Tipo de } \\
\text { afrontamiento }\end{array}$} & \multicolumn{2}{|c|}{ Número de respuestas } \\
\hline & & Mujeres & Hombres \\
\hline \multirow{2}{*}{ Aislamiento } & Afrontamiento activo & 0 & 0 \\
\hline & Afrontamiento pasivo & 4 & 2 \\
\hline \multirow{2}{*}{ Soledad } & Afrontamiento activo & 0 & 0 \\
\hline & Afrontamiento pasivo & 2 & 0 \\
\hline \multirow{2}{*}{ Seguir adelante } & Afrontamiento activo & 2 & 1 \\
\hline & Afrontamiento pasivo & 0 & 0 \\
\hline \multirow{2}{*}{$\begin{array}{l}\text { Participación en } \\
\text { actividades }\end{array}$} & Afrontamiento activo & 9 & 9 \\
\hline & Afrontamiento pasivo & 0 & 0 \\
\hline
\end{tabular}

\section{Discusión}

El presente estudio tuvo como fin comprender la forma en que un grupo de personas mayores colombianas viven $\mathrm{y}$ afrontan la viudez -considerado uno de los eventos más estresantes en la adultez mayor-, así como conocer la relación entre las respuestas psicológicas, el sexo y los estilos de afrontamiento desde un enfoque de metodología mixta y con base en el estudio de Bennett et al. (2005). Este estudio es un acercamiento inicial a este fenómeno en el que se reportan las respuestas psicológicas de un grupo de personas mayores viudas que facilitan el afrontamiento exitoso a la pérdida de la pareja y cuáles lo dificultan.

Los resultados mostraron que existen unas respuestas psicológicas similares a las reportadas por Bennett et al. (2005) en Inglaterra. Aquellas respuestas que se relacionan con mantener activa la red social fueron encontradas en ambos estudios como adaptativas (conseguir pareja, tener amigos de verdad, ayudar a otros y realizar actividades), mientras que respuestas como aislamiento se asociaron con un afrontamiento pasivo. Otras respuestas similares fueron las asociadas al proceso de duelo y afrontamiento como lo son dolor, tristeza, tranquilidad y deprimido.

La teoría ecológica de Bronfenbrenner (1979) destaca que el contexto social distal como el sistema de estratificación social, no afecta a las personas de manera uniforme. Los resultados encontrados en este estudio muestran que el contexto social distal y los recursos y vulnerabilidades inter o intrapersonales proximales son interactivos en la conformación de los factores determinantes del afrontamiento activo y pasivo, en este caso de la pérdida por viudez en la adultez mayor. De este modo el sentido de vida como una característica personal e individual de las personas mayores participantes se encontró como determinante para el afrontamiento, aspecto que no se da de manera única, sino que va de la mano de factores micro-sociales como el apoyo social y las redes con las que se cuenta en la viudez. Asimismo, a nivel macrosocial, se encontró que las dificultades económicas como consecuencia de la viudez y el poco apoyo estatal percibido sobre todo por las mujeres que dependían económicamente de sus esposos, fue un factor importante relacionado con un afrontamiento pasivo o no exitoso. Estos hallazgos concuerdan con lo encontrado por Antonucci et al. (2002) respecto al apoyo del estado y las políticas como factores significativamente influyentes para el afrontamiento exitoso a la viudez.

Las dificultades económicas encontradas en el presente estudio se hallaron como un factor para un afrontamiento pasivo o no exitoso específicamente en mujeres, resultado 
sustentado también por los hallazgos de Ángel et al. (2007). Esto se relaciona directamente con la dependencia económica encontrada sólo en las mujeres. En particular, suplir esta necesidad garantizó un éxito en el afrontamiento para algunas mujeres. Esto lo lograron continuando con el trabajo, buscando el apoyo de hijos o heredando la pensión, lo que particularmente les generó independencia económica y sensación de libertad.

El presente estudio reveló que los hombres en mayor grado que las mujeres, buscaban suplir la dependencia instrumental por medio de la búsqueda de otra pareja, lo que es consistente con lo encontrado por Pérez (2008), quien relata que es más probable que después de perder la pareja, los hombres tengan mayor posibilidad de volver a entablar una relación. En concordancia con Bennett et al. (2005), los hombres buscaron consuelo en la compañía de otras mujeres y apoyo emocional, además de suplir necesidades instrumentales. Otras mujeres se mostraron poco interesadas en buscar nueva pareja, lo que en ambos estudios se relaciona con un sentido de fidelidad adquirido, que para las viudas debe continuar incluso luego de la muerte del cónyuge. Respecto a ello, Bennett (2010) asegura que las mujeres hablan y negocian sus identidades en términos de lucha personal, en donde aún desean mantener el vínculo permanente con su marido.

En cuanto a la fase cuantitativa, en el presente estudio no se encontraron diferencias de sexo respecto a las respuestas psicológicas, resultado que sí fue reportado por Bennett et al. (2005); estas diferencias pueden deberse al tamaño del grupo de participantes de ambos estudios. La ausencia de interacción entre sexo y respuesta psicológica indica que para este estudio no existen respuestas diferenciales por sexo para el tipo de afrontamiento, sólo respuestas psicológicas generales que fueron más adaptativas.

El análisis cuantitativo reveló que sólo dos vías de interacción fueron significativas (afrontamiento*respuesta), lo que concuerda con las categorías centrales encontradas en el análisis a partir de teoría fundamentada, las cuales son el apoyo social y el sentido de vida. Ambas fueron determinantes para el afrontamiento de ambos sexos, tanto en el análisis cualitativo como en el cuantitativo.

Las mujeres que reportaron la ausencia de apoyo social y comentaron respuestas psicológicas como aislamiento y soledad luego de la pérdida de su cónyuge, no han logrado afrontar. Por consiguiente, dicha situación puede deberse a características socioculturales que poseen las mujeres, lo cual las hace más propensas a necesitar estar cerca de otros, conversar y recibir apoyo social, emocional o instrumental, lo que se relaciona con el estudio realizado por Li, Liang, Toler y Gu (2004).

Uno de los aspectos encontrados en el estudio que se informa, no reportados por Bennett et al. (2005), fue que para las personas mayores viudas colombianas resulta decisivo el hecho de evitar una ruptura severa en su cotidianidad y de su identidad, lo que es logrado a través del mantenimiento de los roles desempeñados durante su vida de casados.

Al igual que en el presente estudio, Bennett (2004) encontró que existen factores asociados a la muerte del cónyuge que intervienen en el afrontamiento de la pérdida, entre ellos se encuentra el significado que le dan a la muerte, relacionándolo con las creencias e ideas religiosas, lo cual les ayuda a explicar la muerte y por lo tanto a reducir los sentimientos dolorosos. Adicionalmente, los hallazgos del estudio colombiano revelan que la existencia de la creencia en Dios aleja sentimientos de culpa y ayuda a la resignación y a la expresión de emociones.

Los resultados de este estudio proporcionan sustento a la idea de que el afrontamiento es producto de las interacciones entre los sistemas macro y micro determinantes a través del tiempo y muestra a la viudez como una transición multifacética, abordando las consecuencias individuales supeditadas a las condiciones macro y micro sociales, como las condiciones económicas, el sexo, las redes de 
apoyo, la asignación de roles sociales, incluyendo la calidad del matrimonio, los atributos a nivel individual, pensamientos y creencias.

Por último, esta investigación presenta algunas limitaciones; la primera es haber tenido menos personas con un afrontamiento pasivo, ya que más de la mitad de los participantes había logrado un afrontamiento activo. Ello se relaciona con otro limitante, que fue el no contar con un instrumento de clasificación del tipo de afrontamiento para haber realizado un filtro, con el fin de incluir más personas que no afrontaron exitosamente la pérdida. Sin embargo, se realizó de la misma forma que lo hicieron Bennett et al (2005). De igual forma, en estudios posteriores sería interesante incluir una escala que realice la clasificación.

\section{Referencias bibliográficas}

Ángel, J., Jiménez, M. y Ángel, R. (2007). The economic consequences of widowhood for older minority women. The Gerontologist, 47(2), 224-234.

Antonucci, T., Lansford, J., Akiyama, H., Smith, J., Baltes, M., Keiko, T., Fuhrer, R. y Dartigues, J. (2002). Differences between men and women in social relations, Resource deficits and depressive symptomatology during later life in foundations. Journal of Social Issues, 58(4), 767-783. http://dx.doi.org/10.1111/15404560.00289 .

Bennett, K.M. (2003).Social engagement as a predictor of objective and subjective health. The Gerontologist, 43(2), 557- 563.

Bennett, K.M. (2004). Why did he died? The attributions of cause of death among women widowed in later life. Journal of Health Psychology, 9(3), 345-353. http://dx.doi. org/10.1177/1359105304038242.

Bennett, K.M. (2006). Does marital status and marital status change predict physical health in older adults? Psychological Medicine, 36, 1313-1320.

Bennett, K.M. (2010). You can't spend years with someone and just cast them aside: Augmented identity in older British widows. Journal of Women and Aging, 22(3), 204-217. http:// dx.doi.org/10.1080/08952841.2010.495571.

Bennett, K., Hughes, G. y Smith, P. (2003). I think a woman can take it: Widowed men's views and experiences of gender differences in bereavement. Again International, 23(4), 408-424.

Bennett, K., Hughes, G. y Smith, P. (2005). Psychological response to later life widowhood: coping and the effects of gender. Omega, 51(1), 33-52.

Bennett, K.M. y Soulsby, L. (2012). Wellbeing in bereavement and widowhood. Illness, Crisis $y$ Loss, 24(4), 321-337. doi:http://dx.doi. org/10.2190/IL.20.4.b

Bennett, K.M. y Vidal, S. (2000). Narratives of death: a qualitative study of widowhood in later life. Ageing and Society, 20, 413-428.

Bisconti, T., Bergeman, C. y Boker, S. (2006). Social support as a predictor of variability: An examination of the adjustment trajectories of recent widows. Psychology and Aging, 21(3), 590-599. $\quad$ http://dx.doi.org/10.1037/08827974.21.3.590.

Bonnano, G.A. y Kaltman, S. (1999). Toward an integrative perspective bereavement. Psychological Bulletin, 125, 760-776. http://dx.doi. org/10.1037/0033-2909.125.6.760.

Bonnano, G., Wortman, C. y Nesse, R. (2004). Prospective patterns of resilience and maladjustment during widowhood. Psychology and Aging, 19(2), 260-271.

Bronfenbrenner, U. (1979). The ecology of human development. Cambridge: Harvard University Press.

Bryant, A. y Charmaz, K. (2007). Introduction: grounded theory research: Methods and practices. En A. Bryant y K. Charmaz (Eds.), The SAGE handbook of qualitative research in psychology (pp. 1-27). Thousand Oaks, CA: SAGE.

Carr, D. (2004). Gender, pre-loss marital dependence, and older adults' adjustment to widowhood. Journal of Marriage and the Family, 66, 220-235.

Carr, D., House, J.S., Kessler, R.C., Nesse, R.M., Sonnega, J. y Wortman, C. (2000). Marital 
quality and psychological adjustment to widowhood among older adults: A longitudinal analysis. Journals of Gerontology, Series B -Psychological Sciences and Social Sciences, 55(6), 53-74.

Carr, D., Nesse, R. y Wortman, C. (2006). Spousal bereavement in late life. United States of America: Springer.

Carver, C., Scheier, M. y Weintraub, J. (1989). Assessing coping strategies: A theoretically based approach. Journal of Personality and Social Psychology, 56(2), 267-283.Charmaz, K. (2006). Constructing grounded theory: A practical guide through qualitative analysis. London: SAGE

Choi, H. y Marks, N. (2011). Socioeconomic status, marital status continuity and change, marital conflict, and mortality. Journal of Aging Health, 23(4), 714-742.

Cresswell, J. (2014). Research design: qualitative, quantitative and mixed methods approaches. Thousand Oaks, CA.: SAGE.

Del Risco, P. (2009). Soporte social y satisfacción con la vida en un grupo de adultos mayores que asisten a un programa para el adulto mayor. (Tesis de Licenciatura no publicada). Facultad de Psicología. Perú: Pontificia Universidad Católica del Perú. Recuperado de http:// enterprise.astm.org.ezproxybib.pucp.edu.pe/ repositor io/bitstream/handle/123456789/399/

DEL_RISCO_AIRALDI_PATRICIA_ SOPORTE_SOCIAL_SATISFACCION. pdf? sequence $=1$.

Dupre, M.E. y Meadows, S.O. (2007) Disaggregating the effects of marital trajectories on health. Journal of Family Issues, 28, 623-652.

Fry, P. (1998). Spousal loss in late life: A 1-year follow-up of perceived changes in life meaning and psychosocial functioning following bereavement. Journal of Personal and Interpersonal Loss, 3, 369-391.

Guiaux, M., Van, T. y Broese, M. (2007). Changes in contact and support: exchange in personal networks after widowhood. Personal Relationship, 14(1), 457-473. http://dx.doi. org/10.1111/j.1475-6811.2007.00165.x

Ha, J., Carr, D., Utz, R. y Nesse, R. (2006). Older adults' perceptions of intergenerational support after widowhood how do men and women differ? Journal of Family Issues, 27(1), 3-30. http://dx.doi.org/10.1177/0192513X05277810.

Holm, A. y Severinsson, E. (2012). Systematic review of the emotional state and self- management of widows. Nursing and Health Sciences, 14(1), 109-120. http://dx.doi.org/10.1111/ j.1442-2018.2011.00656.x.

Karraker, A. y De Lamater, J. (2013). Past-year sexual inactivity among older married persons and their partners. Journal of Marriage and Family, 75(1), 142-164. http://dx.doi. org/10.1111/j.1741-3737.2012.01034.x.

Li, L., Liang, J., Toler, A. y Gu, S. (2004). Widowhood and depressive symptoms among older Chinese: do gender and source of support make a difference? Social Science \& Medicine, 60(3), 637-647. http://dx.doi.org/10.1016/j. socscimed.2004.06.014.

Maher, M., Mora, P. y Leventhal, H. (2006). Depression as a predictor of perceived social support and demand: a componential approach using a prospective sample of older adults. Emotion, 6(3), 450-458. http://dx.doi. org/10.1037/1528-3542.6.3.450

Morrow-Howell, N. (2010). Volunteering in later life: research frontiers. Journal of Gerontology: Social Sciences, 65(4), 461-469. doi:10.1093/ geronb/gbq024.

Ocampo, J., Romero, N. y Saa, H. (2001). Prevalencia de las prácticas religiosas, disfunción familiar, soporte social y síntomas depresivos en adultos mayores en Cali, Colombia. Revista Colombiana Médica, 37(2), 26-30.

Pérez, V. (2008). Human sexuality: a look from the older adult's perspective. Revista Cubana de Medicina General, 24(1), 1-8.

Sánchez, P. (2009). Viudedad y vejez: estrategias de adaptación en las personas mayores en España. Madrid: AU Libres.

Schaan, B. (2013). Widowhood and depression among older Europeans -the role of gender, caregiving, marital quality, and regional context. The Journal of Gerontology, 68(3), 431-442. http://dx.doi.org/10.1093/geronb/ gbt015 
Silverstein, M. y Giarrusso, R. (2010). Aging and family life: a decade review. Journal Marriage and Family, 72(1), 1039-1058. http://dx.doi. org/10.1111/j.1741-3737.2010.00749.x

Stimpson, J., Kuo, J., Ray, L., Raji, M. y Peek, K. (2007). Risk of mortality related to widowhood in older Mexican Americans. Annals of Epidemiology, 17(4), 313-319.Strauss, A. y Corbin, J. (2002). Bases de la investigación cualitativa. Técnicas y procedimientos para desarrollar la teoría fundamentada. Universidad de Antioquía: Colombia.

Tashakkori, A. y Creswell, J. (2007). Editorial: The new era of mixed methods. Journal of Mixed Methods Research, 1, 3-6.

Tak-Cheng, S. y Chan, A. (2006). Relationship with others and life satisfaction in later life: do gender and widowhood make a difference? The Journals of Gerontology Series B, 61(1), 46-53.

Vega, D. (2009). Los discursos sobre la calidad de vida de hombres y mujeres mayores, desde una perspectiva de género. (Tesis de maestría no publicada). Facultad de Ciencias Sociales.
Chile: Universidad de Chile. Recuperado de $\quad$ http://www.cybertesis.uchile.cl/tesis/ uchile/2009/cs-vega_d/pdfAmont/csvega_d m.pdf.

Welsh, D., Moore, S. y Getzlaf, A. (2012). Meaning in life. Research in Gerontological Nursing, 5(3), 185-194. http://dx.doi. org/10.3928/19404921-20120605-05

Wilcox, S., Aragaki, A., Mouton, C., Evenson, K., Wassertheil-Smoller, S. y Loevinger, B. (2003). The effects of widowhood on physical and mental health, health behaviors, and health outcomes: The women's health initiative. Health Psychology, 22(5), 513-522. http:// dx.doi.org/10.1037/0278-6133.22.5.513.

Williams, K. y Umberson, D. (2004). Marital status, marital transitions, and health: a gendered life course perspective. Journal of Health Social Behavior, 45, 81-98.

Wruz, C., Hänel, M., Wagner, J., y Neyer, F. (2013). Social network changes and life events across the life span: a meta-analysis. Psychological Bulletin, 139(1), 53-80. http://dx.doi. org/10.1037/a002.

Recibido: 9 de marzo de 2018 Aceptado: 15 de enero de 2020 
